\title{
Small Heat Shock Proteins Induce a Cerebral Inflammatory Reaction
}

\author{
Ilona B. Bruinsma, ${ }^{1,2}$ Mieke de Jager, ${ }^{1,2}$ Anna Carrano, ${ }^{5}$ Alexandra A. M. Versleijen, ${ }^{1,2}$ Robert Veerhuis, ${ }^{6}$ \\ Wilbert Boelens, ${ }^{3}$ Annemieke J. M. Rozemuller, ${ }^{5}$ Robert M. W. de Waal, ${ }^{4}$ and Marcel M. Verbeek ${ }^{1,2}$ \\ Departments of ${ }^{1}$ Neurology and Alzheimer Centre, ${ }^{2}$ Laboratory Medicine, ${ }^{3}$ Biomolecular Chemistry and Nijmegen Centre for Molecular Life Sciences, and \\ ${ }^{4}$ Pathology, Donders Institute for Brain, Cognition and Behaviour, Centre for Neuroscience, Radboud University Nijmegen Medical Centre, 6500 HB \\ Nijmegen, The Netherlands, and Departments of ${ }^{5}$ Neuropathology and ${ }^{6}$ Clinical Chemistry, Psychiatry and Alzheimer Center, VU University Medical \\ Center, 1081 BT Amsterdam, the Netherlands
}

More than $80 \%$ of Alzheimer's disease (AD) patients have some degree of cerebral amyloid angiopathy (CAA). In addition to arteries and veins, capillaries can also be affected. Capillary CAA (capCAA), rather than CAA in larger vessels, is associated with flame-like amyloidbeta $(\mathrm{A} \beta)$ deposits that may extend beyond the vessel wall and radiate into the neuropil, a phenomenon also known as "dyshoric angiopathy." $\mathrm{A} \beta$ deposits in $\mathrm{AD}$, parenchymal as well as (cap)CAA and dyshoric angiopathy, are associated with a local inflammatory reaction, including activation of microglial cells and astrocytes that, among others, produce cytokines and reactive oxygen species. This neuroinflammatory reaction may account for at least part of the cognitive decline. In previous studies we observed that small heat shock proteins (sHsps) are associated with $\mathrm{A} \beta$ deposits in $\mathrm{AD}$. In this study the molecular chaperones Hsp20, HspB8 and HspB2B3 were found to colocalize with CAA and capCAA in AD brains. In addition, Hsp20, HspB8 and HspB2B3 colocalized with intercellular adhesion molecule 1 (ICAM-1) in capCAA-associated dyshoric angiopathy. Furthermore, we demonstrated that Hsp20, HspB8 and HspB2B3 induced production of interleukin 8, soluble ICAM-1 and monocyte chemoattractant protein 1 by human leptomeningeal smooth muscle cells and human brain astrocytes in vitro and that Hsp27 inhibited production of transforming growth factor beta 1 and CD40 ligand. Our results suggest a central role for sHsps in the neuroinflammatory reaction in $\mathrm{AD}$ and $\mathrm{CAA}$ and thus in contributing to cognitive decline.

\section{Introduction}

In Alzheimer's disease $(\mathrm{AD})$ amyloid- $\beta(\mathrm{A} \beta)$ is deposited as plaques in the parenchyma, but also as cerebral amyloid angiopathy (CAA) (Selkoe, 1991). Capillary A $\beta$ deposits (capCAA) correlates with severity of $\mathrm{AD}$ pathology and clinical deterioration, whereas larger vessel CAA does not (Attems and Jellinger, 2004; Eurelings et al., 2010). Furthermore, in capCAA, flame-like A $\beta$ deposits may extend beyond the vessel wall and radiate into the neuropil, a phenomenon known as "dyshoric angiopathy" (Attems et al., 2011). Neuropathological examination of (cap)CAA reveals activated microglia and astrocytes surrounding these lesions (McGeer and McGeer, 1995; Yamada et al., 1996; Akiyama et al., 2000; Rozemuller et al., 2005; Richard et al., 2010; Attems et

\footnotetext{
Received Feb. 21, 2011; revised May 18, 2011; accepted June 23, 2011.

Author contributions: I.B.B. and M.M.V. designed research; I.B.B., M.d.J., A.C., and A.A.V. performed research; R.V., W.C.B., and A.J.M.R. contributed unpublished reagents/analytic tools; I.B.B., R.M.D.W., and M.M.V. analyzed data; I.B.B., R.M.D.W., and M.M.V. wrote the paper.

This study was supported by grants from the Internationale Stichting Alzheimer Onderzoek (ISA0, no. 07510), the Netherlands Organisation for Scientific Research (NW0/ZonMW, Vidi program, no. 917.46.331) and the Hersenstichting Nederland (no. 14F06.18). We thank Dr. D. Schenk for his generous gift of antibodies and Prof. Dr. M. Netea for his generous gift of LPS.

The authors declare no competing financial interests.

Correspondence should be addressed to Dr. I. B. Bruinsma, Department of Neurology, 830 LGEM, Radboud University Nijmegen Medical Centre, P.0. Box 9101, 6500 HB Nijmegen, The Netherlands. E-mail: i.bruinsma@neuro.umen.nl.

DOI:10.1523/JNEUROSCI.0945-11.2011

Copyright $\odot 2011$ the authors $\quad 0270-6474 / 11 / 3111992-09 \$ 15.00 / 0$
}

al., 2011) and colocalization of inflammatory factors such as intercellular adhesion molecule 1 (ICAM-1) (Verbeek et al., 1996).

In $\mathrm{AD}$ brains, an inflammatory reaction may occur in conjunction with $\mathrm{A} \beta$ deposits, characterized by the increased expression of, among others, transforming growth factor $\beta 1$ (TGF- $\beta_{1}$ ) (Wyss-Coray et al., 1997), interleukin (IL) $1 \beta$, IL-8, monocyte chemoattractant protein 1 (MCP-1), ICAM-1 and CD40 ligand (Akiyama et al., 2000; Calingasan et al., 2002; Sokolova et al., 2009; Heneka et al., 2010). This inflammatory reaction may occur in response to the presence of extracellular $\mathrm{A} \beta$ or phagocytosis of $\mathrm{A} \beta$ deposits by microglia (Malm et al., 2010). Inflammation, both directly via interaction of activated glial cells and indirectly via secreted neurotoxic mediators may compromise neuronal function. Indeed, transgenic mice with extensive CAA showed cognitive decline as a result of increased microglial activation and neuroinflammation (Xu et al., 2007), which could be reduced by treatment with the anti-inflammatory drug minocycline, and thereby improved cognition (Fan et al., 2007). Epidemiological studies demonstrated that most patients with CAA-related inflammation show at least a partial clinical improvement to high dose corticosteroids or other immunosuppressive agents (Chung et al., 2011). Thus both human and mouse studies provide evidence for a contribution of $\mathrm{A} \beta$ to $\mathrm{CAA}$-related neuroinflammation and subsequent cognitive decline. However, the neuroinflammatory reaction in $\mathrm{AD}$ patients may be more complex.

In $\mathrm{AD}$, several macromolecules colocalize with plaques and CAA, including small heat shock proteins ( $\mathrm{sHsps)} \mathrm{(Wilhelmus} \mathrm{et}$ 
al., 2006b,c). Previous research showed that expression of $\alpha \mathrm{B}$ crystallin and Hsp27 is increased in reactive astrocytes of $\mathrm{AD}$ brains (Renkawek et al., 1993, 1994; Wilhelmus et al., 2006c). Hsp20 codeposited with $A \beta$ in diffuse and classic plaques and HspB2 with classic plaques and CAA (Wilhelmus et al., 2006c). Moreover, $\mathrm{HspB} 8$ colocalized with classic plaques in $\mathrm{AD}$ and with CAA in hereditary cerebral hemorrhage with amyloidosis of the Dutch type (HCHWA-D) (Wilhelmus et al., 2006b). Furthermore, we observed that these sHsps, but not $\mathrm{A} \beta$, induced IL-6 production in various cerebral cell cultures (Wilhelmus et al., 2009), suggesting that sHsps may play a pivotal role in inducing neuroinflammation. Therefore, we investigated the association of sHsps with (cap)CAA and CAA-associated inflammation in postmortem brain and investigated the effect of selected sHsps on the production of inflammatory factors by cultured human leptomeningeal smooth muscle cells and astrocytes in vitro.

\section{Materials and Methods}

Reagents. Lyophilized $\mathrm{A} \beta_{1-42}$ (95\% pure) and $\mathrm{A} \beta_{1-40}$ (98\% pure) were purchased from Quality Controlled Biochemicals. A $\beta$ with the "Dutch" mutation $\left(22 \mathrm{Glu} \rightarrow \mathrm{Gln}, \mathrm{D}-\mathrm{A} \beta_{1-40}, 96 \%\right.$ pure $)$ was purchased from $21^{\text {st }}$ Century Biochemicals. $\mathrm{D}-\mathrm{A} \beta_{1-40}$ was used as a model peptide, because it causes CAA in hereditary cerebral hemorrhage with amyloidosis of the Dutch type (HCHWA-D) (Davis and Van Nostrand, 1996; Verbeek et al., 1997; Wilhelmus et al., 2007). $\mathrm{A} \beta_{1-42}, \mathrm{~A} \beta_{1-40}$, and D-A $\beta_{1-40}$ were dissolved in 1,1,1,3,3,3-hexafluoro-2 propanol (HFIP) (Sigma-Aldrich), air-dried, and dissolved in DMSO at a concentration of $5 \mathrm{~mm}$. Aliquots of the solutions were stored at $-80^{\circ} \mathrm{C}$.

Autopsy material. Patient selection was based on neuropathological findings at autopsy. Tissue samples were selected for the presence of capCAA (Richard et al., 2010). For immunohistochemistry, tissue samples from the occipital neocortex from $3 \mathrm{AD}$ patients with capCAA (age $76 \pm 12$ years; postmortem delay $5: 53 \pm 2: 09 \mathrm{~h}$ ) were obtained after rapid autopsy and immediately frozen in liquid nitrogen. Informed consent was obtained according to European guidelines. Table 1 provides an overview of the diagnosis, Braak stage, CERAD score, CAA grade, age, postmortem interval (PMI) and gender. Neuropathological evaluation was performed on frozen tissue from occipital pole cortex. CAA score was defined as follows: severe $(+++)$, moderate $(++)$, mild $(+)$ as previously described (Richard et al., 2010). Staging of AD was evaluated according to the criteria established by Braak and Braak and CERAD (Braak and Braak, 1991; Mirra et al., 1991).

Immunohistochemistry. In Table 2 the primary antibodies used in this study are listed. Unless mentioned otherwise, we used biotin-labeled horse anti-mouse antibodies (Vector Laboratories) as the detection antibody for mouse monoclonal antibodies, or biotin-labeled goat-antirabbit antibodies (Vector Laboratories) in the case of rabbit polyclonal antibodies.

Serial cryosections $(4 \mu \mathrm{m})$ were air-dried and fixed in acetone for 5 min. Endogenous peroxidase activity was blocked using acetone containing $0.15 \% \mathrm{H}_{2} \mathrm{O}_{2}$ for $5 \mathrm{~min}$. Sections were preincubated for $30 \mathrm{~min}$ with $20 \%$ animal serum, the type of which was determined by the specific biotin-labeled antibody used. Subsequently, sections were incubated overnight at $4^{\circ} \mathrm{C}$ with primary antibody (Table 2 ), with secondary antibodies for $60 \mathrm{~min}$ at room temperature, and with the avidin-biotin complex according to the manufacturer's description. Between incubation steps, sections were extensively washed using PBS. 3-Amino-9-ethyl carbazole (AEC) was used as a chromogen. After a short rinse in tap water the preparations were incubated with hematoxylin for $1 \mathrm{~min}$ and thoroughly washed with tap water for $10 \mathrm{~min}$. Finally, sections were mounted with Imsol mount (Klinipath). All antibodies were diluted in PBS containing $1 \%$ bovine serum albumin (BSA), which also served as a negative control. At regular intervals, sections were stained with anti-A $\beta$ to allow for topographical alignment of CAA with the stains for sHps and inflammatory factors.

For double immunostaining of $\mathrm{A} \beta$ and/or astrocytes/microglia, sections were stained using the EnVision method as previously described
Table 1. Overview of the origin of astrocyte and HBP cell cultures and tissue sections

\begin{tabular}{|c|c|c|c|c|c|c|c|c|}
\hline & & & & & & Plaque & & \\
\hline Imber & Diagnosis & $\begin{array}{l}\text { Age } \\
\text { (years) }\end{array}$ & $\begin{array}{l}\text { PMI } \\
\text { (h:m) }\end{array}$ & Gender & $\begin{array}{l}\text { NFT stage } \\
\text { (Braak) }\end{array}$ & $\begin{array}{l}\text { score } \\
\text { (CERAD) }\end{array}$ & $\begin{array}{l}\text { CAA } \\
\text { grade }\end{array}$ & Cell type \\
\hline
\end{tabular}

Cell culture

\begin{tabular}{lllllllll}
1 & AD & 84 & $6: 30$ & $\mathrm{~F}$ & V & C & N.D. & Astrocyte \\
2 & AD & 92 & $7: 00$ & $\mathrm{~F}$ & $\mathrm{~V}$ & 0 & N.D. & Astrocyte \\
3 & Control & 76 & $4: 40$ & $\mathrm{~F}$ & $\mathrm{I}$ & $\mathrm{B}$ & N.D. & Astrocyte \\
4 & AD & 77 & $3: 00$ & $\mathrm{M}$ & $\mathrm{VI}$ & $\mathrm{C}$ & ++ & SMC \\
5 & Parkinson & 91 & $4: 00$ & $\mathrm{~F}$ & $\mathrm{~V}$ & $\mathrm{C}$ & +++ & SMC \\
6 & Control & 83 & $4: 00$ & $\mathrm{~F}$ & $\mathrm{III}$ & $\mathrm{B}$ & - & $\mathrm{SMC}$ \\
$\mathrm{HC}$ & & & & & & & & \\
7 & AD + CAA & 65 & $7: 20$ & $\mathrm{M}$ & $\mathrm{V}$ & $\mathrm{C}$ & +++ N.A. \\
8 & AD + CAA & 89 & $6: 55$ & $\mathrm{~F}$ & $\mathrm{~V}$ & $\mathrm{C}$ & +++ N.A. \\
9 & AD + CAA & 74 & $3: 25$ & $\mathrm{M}$ & $\mathrm{VI}$ & $\mathrm{C}$ & +++ N.A. \\
10 & Control & 81 & $3: 45$ & $\mathrm{M}$ & $\mathrm{I}$ & 0 & - & N.A. \\
11 & Control & 71 & $6: 05$ & $\mathrm{~F}$ & $\mathrm{III}$ & $\mathrm{C}$ & - & N.A. \\
12 & Control & 92 & $6: 25$ & $\mathrm{~F}$ & $\mathrm{III}$ & $\mathrm{A}$ & + & N.A. \\
\hline
\end{tabular}

Overview of the cases used in this study. IHC, Tissue used for immunohistochemistry; F, female; M, male; N.D., not determined; N.A., not applicable. Grading of AD (Braak scores) was performed as described in Materials and Methods.

Table 2. Primary antibodies used in this study

\begin{tabular}{lllll}
\hline $\begin{array}{l}\text { Primary } \\
\text { antibody }\end{array}$ & Antigen & $\begin{array}{l}\text { Species } \\
\text { raised in }\end{array}$ & Dilution & Source (reference) \\
\hline 6C6 & A $\beta$ & Mouse & $1: 250$ & Dr. Schenk, Elan Pharmaceuticals \\
GFAP & GFAP & Mouse & $1: 10$ & Monosan, Sanbio \\
LN3 & HLA-DR & Mouse & $1: 50$ & eBioscience \\
MAB208 & IL-8 & Mouse & $1: 100$ & R\&D Systems \\
LS-C8642 & MCP-1 & Mouse & $1: 100$ & Lifespan Biosciences \\
CL203 & ICAM-1 & Mouse & $1: 500$ & (Verbeek et al., 1994a) \\
PN-E12 & ICAM-1 & Mouse & $1: 25$ & (Verbeek et al., 1994a) \\
Pab-aBcr & $\alpha$ B-Crystallin & Rabbit & $1: 500$ & (den Engelsman et al., 2003) \\
1426 & Hsp27 & Rabbit & $1: 2000$ & Abcam \\
VDK-p20 & Hsp20 & Rabbit & $1: 4000$ & (van de Klundert et al., 1998) \\
J92 & HspB8 & Rabbit & $1: 2000$ & (Verschuure et al., 2003) \\
F34 & HspB2 & Rabbit & $1: 2000$ & (Verschuure et al., 2003) \\
\hline
\end{tabular}

(Richard et al., 2010). Briefly, sections were incubated overnight at $4^{\circ} \mathrm{C}$ with primary antibodies (Table 2), followed by incubation with EnVision HRP. EnVision 3,3-diaminobenzidine (DAB) was used as chromogen. Antibodies were diluted in PBS/1\% BSA, which also served as a negative control. After each incubation, slides were extensively washed with PBS. For the detection of fibrillar $\mathrm{A} \beta$ in plaques and CAA, sections were stained with Congo Red $(0.5 \%$ in Ethanol $80 \%, 3 \% \mathrm{NaCl})$ for $20 \mathrm{~min}$ at room temperature. Finally, sections were mounted with DePeX mounting medium Gurr.

Expression and purification of recombinant proteins. Recombinant human $\alpha \mathrm{B}$-crystallin and $\mathrm{HspB} 2 \mathrm{~B} 3$ were expressed and purified as described previously (Wilhelmus et al., 2006a). Since it was demonstrated that HspB2 and HspB3 form functional heterogeneous complexes of 150 $\mathrm{kDa}$ (Sugiyama et al., 2000), both proteins were expressed together in one vector. Coding sequences for the following sHsps were ligated into the pET-46 Ek/LIC vector which also introduces a N-terminal His-tag sequence (Novagen, Merck Chemicals Ltd): human Hsp27, human Hsp20, and human HspB8. Hsp20 and HspB8 were expressed in Rosetta(DE3)pLysS competent cells (Novagen). Hsp27 was expressed in its BL21(DE3)pLysS competent cells (Novagen). A culture inoculated from a single colony was induced by addition of $0.25 \mathrm{~mm}$ isopropyl-thio$\beta$-D-galactopyranoside (IPTG) for $4 \mathrm{~h}$ at $37^{\circ} \mathrm{C}$, with the exception of Hsp20, which was induced for $16 \mathrm{~h}$ at $25^{\circ} \mathrm{C}$. After incubation with IPTG, cells were harvested and lysed by sonication in native lysis buffer (NLB; $50 \mathrm{~mm} \mathrm{NaH}_{2} \mathrm{PO}_{4}, 300 \mathrm{~mm} \mathrm{NaCl}, \mathrm{pH}$ 8.0) supplemented with EDTA-free protease inhibitor (Roche Applied Science). Insoluble proteins were removed by centrifugation. All sHsps were purified over a Ni-NTA agarose 
column (Qiagen) using NLB supplemented with increasing concentrations of imidazole (Sigma-Aldrich Chemie BV) ranging from 20 up to $250 \mathrm{~mm}$. After desalting, sHsps were concentrated in $10 \mathrm{~mm}$ Tris, $50 \mathrm{~mm}$ $\mathrm{NaCl}, \mathrm{pH} 7.4$ buffer by using an iCON concentrator spin column (Thermo Fisher Scientific Inc.). Protein concentration was determined using a BCA Protein Assay kit according to the manufacturer's description (Thermo Fisher Scientific). Purified sHsps were aliquoted and stored at $-80^{\circ} \mathrm{C}$. Purity of the preparations was $\geq 95 \%$.

Cell culture. Smooth muscle cells (SMCs) and astrocytes were both isolated from human brain tissue obtained after rapid autopsy, as described previously (De Groot et al., 1997; Verbeek et al., 1994b; Veerhuis et al., 1998) (Table 1). Diagnosis and grading of patients was performed according to the criteria mentioned previously. SMCs were maintained in Eagle's modification of essential medium (EMEM; PAA Laboratories $\mathrm{GmbH}$ ), supplemented with 10\% human serum (Lonza BioWhittaker Benelux BV), 20\% fetal bovine serum (PAA Laboratories GmbH), recombinant basic fibroblast growth factor $(1 \mathrm{ng} / \mathrm{ml})$ and gentamycin $(2.5$ $\mu \mathrm{g} / \mathrm{ml})$. Astrocytes were maintained in a mixture $(1: 1 \mathrm{v} / \mathrm{v})$ of DMEM and Ham's F10 supplemented with 10\% fetal bovine serum, $2 \mathrm{~mm}$ L-glutamine (Cambrex) and gentamycin $(2.5 \mu \mathrm{g} / \mathrm{ml})$. All primary cell cultures were cultured at $37^{\circ} \mathrm{C}, 5 \% \mathrm{CO}_{2}$ and $90 \%$ relative humidity. Although the cultured astrocytes and SMCs were derived from different clinical backgrounds (i.e., either AD or control patients), we did not observe differences with respect to their responses in the various types of experiments.

Cell passages 3-9 were used for the experiments. Control cells incubated with EMEM or DMEM/Ham's F10 and 0.1\% BSA (serum-free medium) demonstrated normal morphology. Experiments were performed at least twice.

ELISA. Duplicate wells (12-well plate; Corning Inc) with cultured cells were preincubated with serum-free medium for a minimum of $4 \mathrm{~h}$. Subsequently, cells were incubated with $12.5 \mu \mathrm{M}$ synthetic $\mathrm{A} \beta_{1-40}, \mathrm{~A} \beta_{1-42}$, D-A $\beta_{1-40}$ or $12.5 \mu \mathrm{M}$ purified sHsps ( $\alpha$ B-crystallin, Hsp27, Hsp20, $\mathrm{HspB} 8$ and $\mathrm{HspB} 2 \mathrm{~B} 3$ ) at $37^{\circ} \mathrm{C}$ and $5 \% \mathrm{CO}_{2}$. This concentration was the lowest concentration to induce a significant effect across all sHsps tested. In addition, the same concentration of $A \beta$ induced biological effects in cultured SMCs and astrocytes (Verbeek et al., 1997; Bruinsma et al., 2010) and was therefore chosen in the experiments. Lipopolysaccharide (LPS; 055:B5; Sigma-Aldrich) stimulation $(1 \mu \mathrm{g} / \mathrm{ml})$ was used as a positive control for inducing cytokine production. After $48 \mathrm{~h}$ of incubation, supernatants and cell lysates (solubilized in RIPA; $50 \mathrm{~mm}$ Tris $\mathrm{HCl}, \mathrm{pH} 7.4,150 \mathrm{~mm} \mathrm{NaCl}, 1 \%$ Nonidet P-40, 0.5\% sodium deoxycholate, and $0.1 \%$ SDS in Milli-Q) were collected, centrifuged at 13.200 rpm for $10 \mathrm{~min}$ at $4^{\circ} \mathrm{C}$ and stored at $-80^{\circ} \mathrm{C}$ until further use. To exclude endotoxin contamination, potentially present in the purified sHsp samples, coincubation with polymixin B (PMB; Sigma-Aldrich; $10 \mu \mathrm{g} /$ $\mathrm{ml}$ ) was used to inhibit LPS-mediated inflammation. In these experiments, PMB was preincubated with both the LPS and the sHsps for $1 \mathrm{~h}$ at $37^{\circ} \mathrm{C}$ before addition to the cells.

Il-8, ICAM-1, MCP-1, CD40 ligand, and TGF- $\beta_{1}$ levels in cell culture supernatant were measured using commercial enzyme immunoassay kits according to the manufacturer's instructions (DuoSet ELISA, R\&D Systems). To correct for differences in cell density, inflammatory factor production was normalized to protein content of the cell lysate which was determined by using a BCA Protein Assay kit according to the manufacturer's description (Thermo Fisher Scientific Inc.). Concentrations of the various inflammatory factors were expressed as $\mathrm{pg} / \mathrm{mg}$ protein.

Statistical analysis. Statistical analyses were performed using an independent sample $t$ test in SPSS 14.0 for Windows software. Statistical significance was set at $p<0.05$.

\section{Results}

Inflammatory cells in $\mathrm{AD}$ brains with severe CAA pathology, capCAA, and dyshoric angiopathy

Double staining for GFAP and Congo red demonstrated the presence of astrocytes around $\mathrm{A} \beta$-laden vessels, with a more prominent GFAP staining around the larger vessels (Fig. $1 A, C$ ). Clusters of HLA-DR-positive microglia were also observed around larger ves-
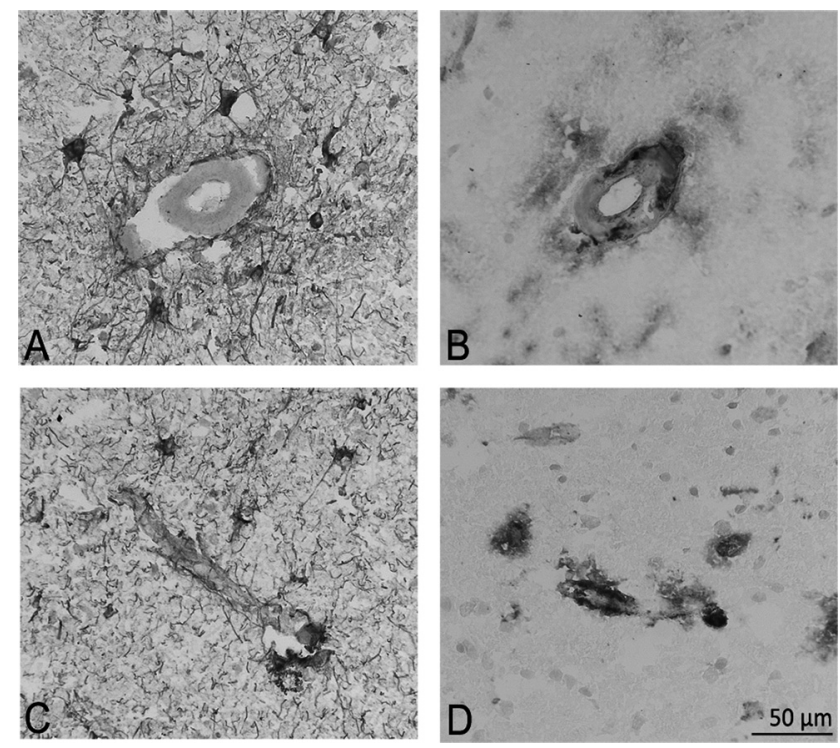

Figure 1. Immunohistochemical staining of $\operatorname{GFAP}(\boldsymbol{A}, \boldsymbol{C})$ and microglial cell marker (LN3; $B$, D) in CAA $(\boldsymbol{A}, \boldsymbol{B})$ and $\operatorname{capCAA}(\boldsymbol{C}, \boldsymbol{D})$. All sections are double stained with Congo Red. GFAPpositive reactive astrocytes are present around both larger vessels and capillaries $(\boldsymbol{A}, \boldsymbol{C})$. Microglial activation is associated with both large vessels and capillaries, however more prominent around the latter $(\boldsymbol{B}, \boldsymbol{D})$.

sels and capillaries laden with $\mathrm{A} \beta$ and were particularly prominent around the capillaries (Fig. 1B,D).

\section{Expression of sHsps in AD brains with severe CAA pathology, capCAA, and dyshoric angiopathy}

In control brains, $\alpha \mathrm{B}$-crystallin staining was observed in glial cells (Fig. 2B). Furthermore, leptomeningeal vessels as well as a few astrocytes in the white matter were also stained (data not shown). Hsp27 staining in control brains was limited to leptomeningeal vessels (data not shown), but not observed in parenchymal vessels (Fig. 2D). Hsp20 staining in control brains was occasionally observed in astrocytes in white and gray matter (data not shown). Weak immunoreactivity of Hsp20 was also observed in large parenchymal (Fig. $2 F$, open arrow) and leptomeningeal vessels (data not shown). HspB8 staining in control brains was observed in astrocytes in both gray and white matter (Fig. $2 \mathrm{H}$, arrow) and in cerebrovascular cells of large parenchymal vessels (Fig. $2 \mathrm{H}$, open arrow). Furthermore, weak staining for HspB2 was observed in cerebrovascular cells in large parenchymal vessels (Fig. $2 J$, open arrow) and in an occasional astrocyte or microglial cell in the brain parenchyma (data not shown). IL-8 (Fig. 2L) and MCP-1 (Fig. 2P) staining was not observed in normal parenchymal or leptomeningeal vessels in control brains, whereas ICAM-1 staining was observed in endothelial cells in control brains (Fig. $2 N$, arrow).

In $\mathrm{AD}$ brains with severe (cap)CAA pathology, the anti- $\mathrm{A} \beta$ antibody (6C6) stained $\mathrm{A} \beta$-affected vessels and capillaries (Fig. $3 A, B$ ). In addition, dyshoric angiopathy (Fig. $3 A$ ) was often observed. The antibodies directed against $\alpha \mathrm{B}$-crystallin, Hsp27, Hsp20, HspB8 and HspB2 showed different staining patterns for these sHsps in CAA, capCAA and dyshoric angiopathy. Representative staining results are shown in Figure 3 and a summary of the immunohistochemical results is shown in Table 3. Anti- $\alpha \mathrm{B}$ crystallin antibody stained reactive astrocytes and activated microglia associated with CAA (Fig. 3C) and capCAA (Fig. 3D). Hsp27 staining was not observed in gray matter (Fig. $3 E, F$ ), but was occasionally observed in reactive astrocytes and microglia in 
Anti-A $\beta$
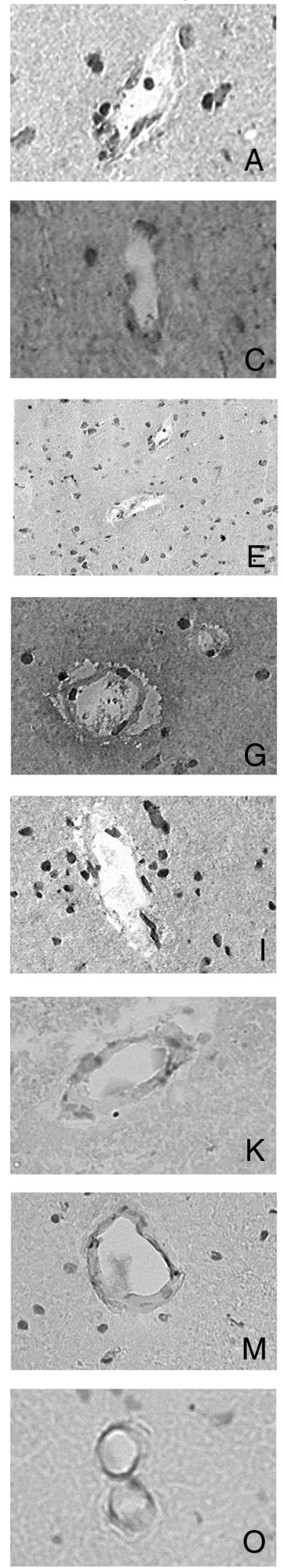
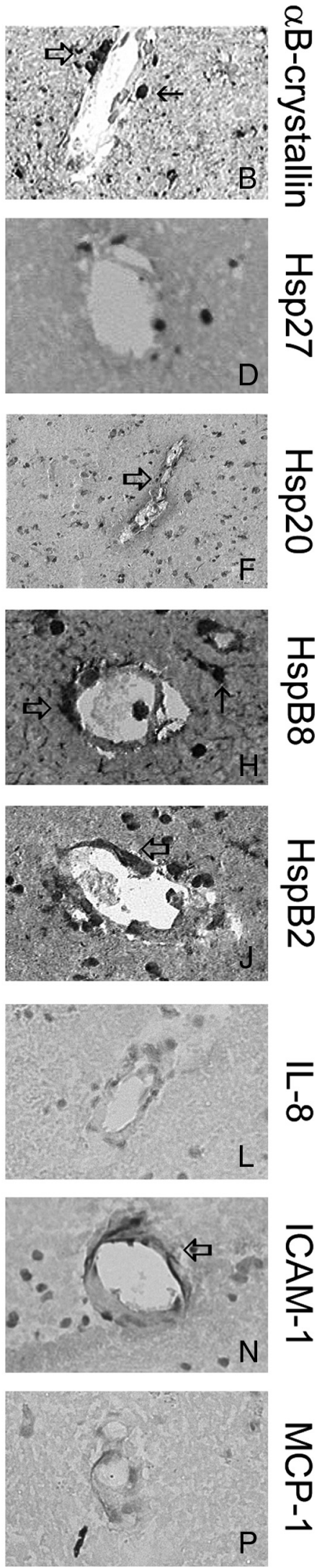

Figure 2. Immunohistochemical staining of $A \beta(A, C, E, G, I, K, M, O)$, $\operatorname{sHsps}(B, D, F, H, J$; open arrow), and inflammatory factors $(\boldsymbol{L}, \boldsymbol{N}, \boldsymbol{P}$; open arrow) in or near normal vessels in the occipital cortex of control patients. Serial sections: $A, B ; C, D ; E, F ; G, H ; I, J ; K, L ; M, N ; O, P$. Anti- $\alpha$ B-crystallin antibodies stained glial cells throughout the cortex ( $\boldsymbol{B}$, arrow). Anti-Hsp27

the white matter (data not shown). In addition, reactive astrocytes surrounding CAA were immunopositive for Hsp20 (Fig. $3 G$ ) and occasionally $\mathrm{Hsp}$-20-positive reactive astrocytes were observed surrounding capCAA (data not shown). Anti-HspB8 stained reactive astrocytes and microglia in both white and gray matter and occasionally immunoreactivity of $\mathrm{HspB} 2$ was observed with reactive astrocytes and microglia. Whereas HspB2 (Fig. $3 L$ ) colocalized with $\mathrm{A} \beta$ in capCAA and CAA, no immunostaining for $\alpha \mathrm{B}$-crystallin (Fig. 3C,D), Hsp27 (Fig. $3 E, F$ ), Hsp20 (Fig. 3G,H) and HspB8 (Fig. 3I,J) was observed. Furthermore, colocalization of Hsp20 (Fig. 3G), HspB8 (Fig. 3I) and HspB2 (Fig. 3K), but not of $\alpha \mathrm{B}$-crystallin (Fig. 3C) and Hsp27 (Fig. 3E), with $\mathrm{A} \beta$ surrounding $\mathrm{CAA}$ and capCAA (dyshoric angiopathy) was observed.

Expression of inflammatory factors in $\mathrm{AD}$ brains with severe CAA pathology, capCAA, and dyshoric angiopathy

ICAM- 1 was associated with dyshoric $A \beta$ deposits surrounding $\mathrm{CAA}$ and capCAA, but not with the $\mathrm{A} \beta$ deposits within the vessel wall (Fig. 3O,P), suggesting colocalization of Hsp20, HspB8 and HspB2 with ICAM-1 in dyshoric angiopathy near (cap)CAA. Although ICAM-1 has also been detected in a soluble form (Harning et al., 1991; Rothlein et al., 1991), it is mainly described as a membrane-bound cell adhesion molecule. Therefore, the ICAM-1 staining we observed is very likely due to membranebound ICAM-1. In earlier studies an increase in IL- 8 and MCP-1 in $\mathrm{AD}$ brains was observed after soluble protein extraction of human brain lysates or in paraffin-embedded formalin-fixed tissue (Akiyama et al., 2000; Calingasan et al., 2002; Sokolova et al., 2009). In the present study, no IL- 8 and MCP- 1 was detectable in tissue from $\mathrm{AD}$ patients with severe CAA pathology (Fig. $3 M, N, Q, R)$. In cryosections, these soluble factors might escape detection due to mild fixation of the tissue.

\section{sHsps induce the production of inflammatory factors by} human leptomeningeal smooth muscle cells and human brain astrocytes

Our immunohistochemical studies suggest that both $\mathrm{A} \beta$ and sHsps accumulate in or near (cap)CAA, where an inflammatory reaction is observed as well. Since human leptomeningeal SMCs are already early affected by A $\beta$ in CAA (Vinters et al., 1996), we studied whether $\mathrm{A} \beta$ or sHsps were able to induce production of cytokines and other inflammatory factors by cultured SMCs. In addition, since reactive astrocytes colocalize near (cap)CAA (Fig. 1 ), we also studied the potential of $\mathrm{A} \beta$ and sHsps to induce an inflammatory reaction in human brain astrocytes.

Neither $\mathrm{A} \beta_{1-40}, \mathrm{~A} \beta_{1-42}$, nor D-A $\beta_{1-40}(12.5 \mu \mathrm{M})$ induced production of IL-8, ICAM-1, MCP-1, TGF- $\beta_{1}$ or CD40 ligand by SMCs or astrocytes, above control levels (Figs. 4, 5). Incubation of $1 \mu \mathrm{g} / \mathrm{ml}$ LPS induced IL- 8 production (Fig. $4 A$; $p<0.05$ ) by SMCs, but not production of soluble ICAM-1 (sICAM-1; Fig.

antibodies were only occasionally immunoreactive with leptomeningeal vessels (data not shown), but not with parenchymal vessels (D). Hsp20 was occasionally demonstrated in astrocytes in normal brains (data not shown). Weak immunoreactivity of Hsp20 was also observed in large parenchymal $(\boldsymbol{F}$, open arrow) and leptomeningeal vessels. HspB8 staining was observed in astrocytes in both gray and white matter $(\boldsymbol{H}$, arrow) and in cerebrovascular cells of large parenchymal vessels ( $\boldsymbol{H}$, open arrow). Weak staining for $\mathrm{HspB} 2$ was observed in cerebrovascular cells in large parenchymal vessels ( $\boldsymbol{J}$, open arrow) and in an occasional astrocyte or microglial cell in the brain parenchyma (data not shown). Furthermore, anti-IL-8 $(\boldsymbol{L})$ and anti-MCP-1 (P) staining was absent from normal parenchymal or leptomeningeal vessels. The anti-ICAM antibodies stained endothelial cells ( $\boldsymbol{N}$, arrow). Original magnification $200 \times$. 
$4 B$ ), MCP-1 (Fig. 4C) and TGF- $\beta_{1}$ (Fig. $4 D)$. Furthermore, incubation of $1 \mu \mathrm{g} / \mathrm{ml}$ LPS induced IL-8 (Fig. $5 A ; p<0.01$ ), sICAM-1 (Fig. $5 B ; p<0.01$ ) and MCP-1 (Fig. $5 C$; $p<0.05$ ) production by astrocytes, but not production of TGF- $\beta_{1}$ (Fig. 5D) and CD40 ligand (Fig. 5E). After preincubation and coincubation of LPS with $\mathrm{PMB}$, production of inflammatory factors by SMCs and astrocytes returned to control levels (Fig. 5; $p<0.05$ ), indicating that PMB efficiently inhibited the LPSinduced inflammatory reaction.

Incubation of SMCs with $12.5 \mu \mathrm{M}$ Hsp20, HspB8 or HspB2B3 resulted in a strong induction of IL-8 (Fig. $4 A ; p<0.01$ ), sICAM-1 (Fig. 4B; Hsp20, HspB2B3, $p<$ 0.001 ; HspB8, $p<0.01$ ) and MCP-1 (Fig. $4 C$; Hsp20, HspB2B3, $p<0.001$; HspB8, $p<0.01$ ) secretion, but not of TGF- $\beta_{1}$ (Fig. $4 D)$. In contrast to the increased levels of IL-8 production after incubation with 12.5 $\mu \mathrm{M}$ Hsp27 (Fig. $4 A ; p<0.001$ ), decreased $(p<0.01)$ TGF- $\beta_{1}$ production was observed after Hsp27 treatment compared with control (Fig. 4D). In addition, incubation with Hsp27 did not induce levels of sICAM-1 (Fig. 4B) and MCP-1 (Fig. 4C) above control levels in SMCs. Furthermore, SMCs did not seem to secrete CD40 ligand, since after incubation of these cells with either $\mathrm{A} \beta_{1-40}, \mathrm{~A} \beta_{1-42}$, and $\mathrm{D}-\mathrm{A} \beta_{1-40}$ or sHsps, the concentrations of CD40 ligand remained below the detection level (data not shown).

Incubation of astrocytes with $12.5 \mu \mathrm{M}$ Hsp20, HspB8 or HspB2B3 also resulted in a strong induction of IL-8 (Fig. $5 A ; p<$ 0.001 ), sICAM-1 (Fig. 5B; $p<0.001$ ) and MCP-1 (Fig. $5 C ; p<0.001$ ) secretion, but not of TGF- $\beta_{1}$ (Fig. 5D). In addition, only $12.5 \mu \mathrm{M}$ HspB8 and HspB2B3 induced CD40 ligand production by astrocytes (Fig. $5 E ; p<0.05$ and $p<0.01$ respectively). Comparable to the effects of Hsp27 in SMCs, IL-8 production by astrocytes was also increased after incubation with $12.5 \mu \mathrm{M}$ Hsp27 (Fig. 5A; $p<0.01$ ), however, Hsp27 induced a decrease in the production of TGF- $\beta_{1}$ (Fig. $5 D ; p<0.001$ ) and CD40 ligand (Fig. 5E; $p<0.01$ ) compared with control. Furthermore, incubation with Hsp27 did not result in levels of sICAM-1 (Fig. 5B) and MCP-1 (Fig. 5C) above control levels in astrocytes.

To exclude LPS contamination, potentially present in the purified sHsp samples, we coincubated cell samples with PMB and sHsps to inhibit potential LPS-mediated inflammation. After preincubation and coincubation of LPS with PMB, production of inflammatory factors by SMCs and astrocytes returned to control levels (Fig. $6 A, B ; p<0.05$ ), indicating that PMB efficiently inhibited the LPS-induced inflammatory reaction. Whereas 12.5 $\mu \mathrm{M} \alpha \mathrm{B}$-crystallin seemed to induce IL- 8 secretion by SMCs and astrocytes, this effect was completely abolished by preincubation and coincubation with PMB (Fig. $6 A, B ; p<0.05$ ), indicating that traces of LPS in the $\alpha \mathrm{B}$-crystallin preparations are responsible for the observed effects. In contrast, PMB had no, or only a minimal effect,
Table 3. Summary of the immunohistochemical results

\begin{tabular}{lccc}
\hline & capCAA & CAA & Dyshoric angiopathy \\
\hline$\alpha$ B-crystallin & $0 \%$ & $0 \%$ & $0 \%$ \\
Hsp27 & $0 \%$ & $0 \%$ & $0 \%$ \\
Hsp20 & $10 \%$ & $0 \%$ & $90 \%$ \\
HspB8 & $10 \%$ & $0 \%$ & $100 \%$ \\
HspB2 & $80 \%$ & $100 \%$ & $95 \%$ \\
\hline
\end{tabular}

Quantification of the percentages of $\mathrm{A} \beta$ deposits that are stained by the $\mathrm{H} H \mathrm{sp}$ antibodies in all patients (average of $n=3$ patients)

on the production levels of IL- 8 by SMCs and astrocytes induced by the other sHsps samples (Fig. $6 A, B$ ), suggesting a strong inducing effect of these sHsps on cytokine production.

\section{Discussion}

The main findings of this study are as follows: 1) Hsp20, HspB8 and HspB2 are associated with (cap)CAA and dyshoric angiopathy in $\mathrm{AD}$ brains with severe CAA pathology; 2) these sHsps colocalize 
A
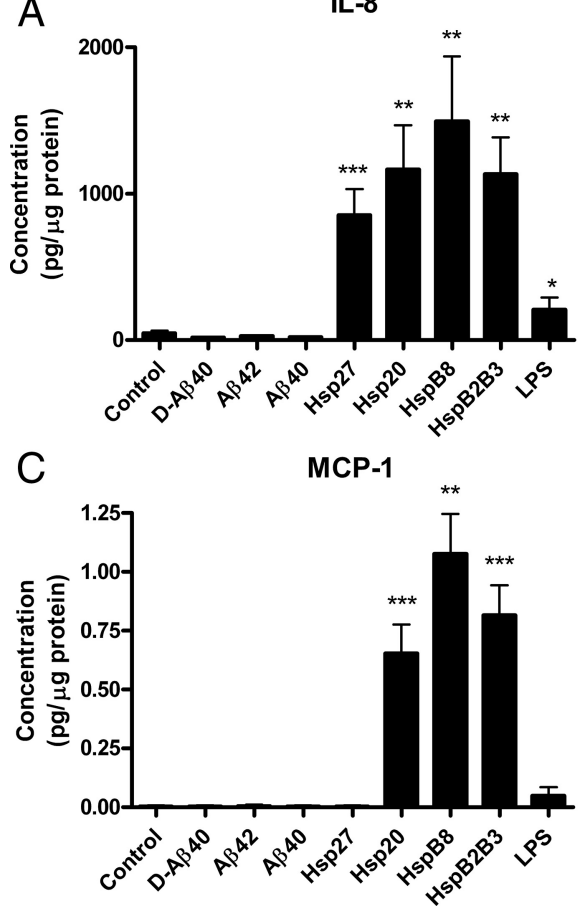

B
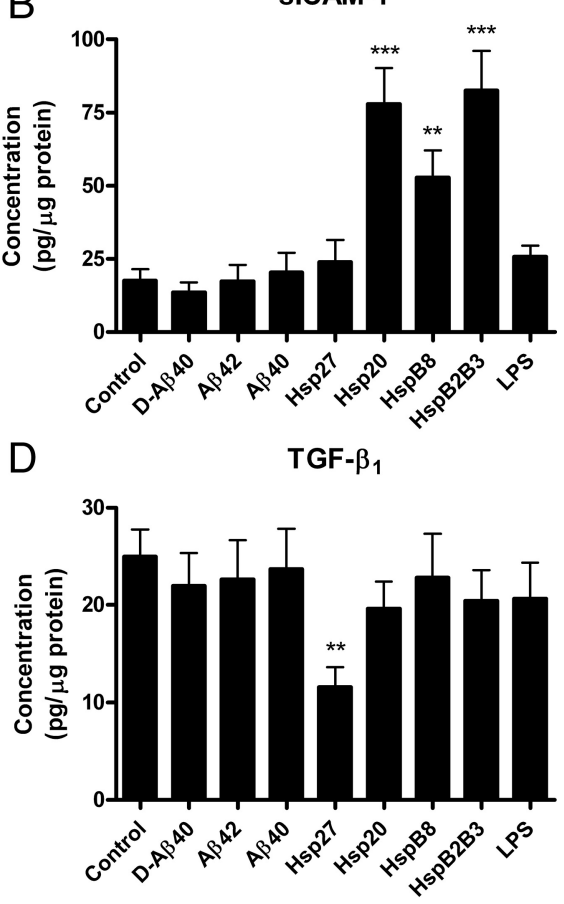

Figure 4. Production of inflammatory factors by human brain leptomeningeal SMCs. Cultured SMCs were incubated with 12.5 $\mu \mathrm{M} \mathrm{D}-\mathrm{A} \beta_{40}, \mathrm{~A} \beta_{42}, \mathrm{~A} \beta_{40}, \mathrm{Hsp27}, \mathrm{Hsp20}, \mathrm{HspB} 8, \mathrm{HspB} 2 \mathrm{~B} 3$, or $1 \mu \mathrm{g} / \mathrm{ml} \mathrm{LPS}$ for $2 \mathrm{~d}$ at $37^{\circ} \mathrm{C}$. Supernatant was collected and IL-8 (A), sICAM-1 (B), MCP-1 (C), and TGF- $\beta_{1}(\boldsymbol{D})$ concentrations were measured using ELISA. Statistical analysis was performed using an independent sample $t$ test. The level of significance of the difference compared with control is indicated as follows: ${ }^{*} p<0.05$; ${ }^{* *} p<0.01 ;{ }^{* * *} p<0.001 ; p>0.05$ is not indicated. Means \pm SEM are shown.

with ICAM-1 in dyshoric angiopathy near (cap)CAA; 3) sHsps (especially Hsp20, HspB8 and $\mathrm{HspB} 2 \mathrm{~B} 3$ ), but not $\mathrm{A} \beta$, increased secretion of IL-8, sICAM-1 and MCP-1 by cultured human leptomeningeal SMCs and human brain astrocytes; 4) Hsp27 reduced secretion of TGF- $\beta_{1}$ by SMCs and astrocytes and CD40 ligand secretion by astrocytes.

The observed astrocytic and microglial activation, indicative of an inflammatory reaction, around (cap)CAA in this report is in line with earlier studies (Richard et al., 2010; Attems et al., 2011). In addition, colocalization of Hsp20, HspB8 and HspB2, but not $\alpha \mathrm{B}$-crystallin and Hsp27, with (cap)CAA and dyshoric angiopathy near (cap)CAA is also in line with our previous studies in which colocalization of these sHsps was found with CAA and/or parenchymal deposits of $A \beta$ (Wilhelmus et al., 2006b,c, 2009). It has been suggested that Hsp20, HspB8 and HspB2 are predominantly associated with fibrillar $\mathrm{A} \beta$ in vivo (Wilhelmus et al., 2006 b,c, 2009) and our results suggest a similar association of these sHsps with fibrillar $\mathrm{A} \beta$ in (cap)CAA and with dyshoric angiopathy near (cap)CAA. Thus, Hsp20, HspB8 and HspB2 are clearly associated with various types of $\mathrm{A} \beta$ deposits in $\mathrm{AD}$ brains and may participate in the local inflammatory reaction.

CAA-related inflammation is of clinical importance since patients with this type of pathology present with cognitive decline, seizures and headaches, that improve upon anti-inflammatory treatment (Eng et al., 2004; Kinnecom et al., 2007; Chung et al., 2011). In addition, it has been suggested that especially capCAA with spreading of the $\mathrm{A} \beta$ deposits into the neuropil could contribute to a rapid clinical deterioration (Eurelings et al., 2010), suggesting an important role of capCAA and dyshoric angiopathy, rather than plaques and CAA, in cognitive decline. In $\mathrm{AD}, \mathrm{A} \beta$ has been shown to trigger neuroinflammation by e.g., activation of the complement system (Heneka et al., 2010), and activation of microglia and astrocytes (Tan et al., 1999; Heneka et al., 2010). In addition, the proinflammatory factors IL-8, MCP-1 and CD40 ligand are increased in $\mathrm{AD}$ (Akiyama et al., 2000; Calingasan et al., 2002; Sokolova et al., 2009) and ICAM-1 and TGF- $\beta_{1}$ colocalize with $\mathrm{A} \beta$ deposits (Verbeek et al., 1996; Wyss-Coray et al., 1997), suggesting that $A \beta$ may induce the expression of these inflammatory factors. In contrast, however, the results of the present study suggest that sHsps may be much more potent inducers of an inflammatory reaction in $\mathrm{AD}$ brains than $\mathrm{A} \beta$. Interestingly, the proinflammatory effect of these sHsps does not seem to be limited to a single parameter such as IL-6 (Wilhelmus et al., 2009), but includes multiple inflammatory factors that are observed in vivo. We obtained in vivo evidence for the colocalization of sHsps near sites of $\mathrm{A} \beta$ associated inflammation in the cerebral vasculature and demonstrated in vitro that Hsp20, HspB8 and HspB2B3 induced production and secretion of IL- 8, MCP-1 and sICAM-1 in SMCs and astrocytes, with little or no effect of Hsp27 and no effect of $\alpha \mathrm{B}$-crystallin on these factors. These results are in line with, and extend, our previous findings that sHsps may be among the key mediators of the inflammatory reactions associated with (cap)CAA, since they also induced IL-6 production in cultured SMCs, astrocytes and microglia (Wilhelmus et al., 2009). Interestingly, our results suggest that those sHsps that occur extracellularly in plaques or dyshoric angiopathy and (cap)CAA (i.e., Hsp20, HspB8 and HspB2B3) have a potent proinflammatory effect, indicating that especially these sHsps are important in the inflammatory reaction in CAA. Interestingly, HspB8 and HspB2B3 induced CD40 ligand expression in astrocytes, but not in SMCs, suggesting that the reported upregulation of CD40 ligand in $\mathrm{AD}$ (van der Wal et al., 1993; Calingasan et al., 2002) might be due to astrocytic production. In contrast to these findings, however, Hsp20, HspB8 and HspB2B3 did not induce production of TGF- $\beta_{1}$ in both SMCs and astrocytes, although these factors are also reported to be upregulated in AD (van der Wal et al., 1993; Calingasan et al., 2002). This suggests that this cytokine might be upregulated by alternative mechanisms or cell types.

Hsp27 is a member of the sHsp family with remarkable capacities compared with the other sHsps tested in this study. Hsp27 was able to induce IL- 8 and inhibit TGF- $\beta_{1}$ production in both human SMCs and astrocytes in vitro. Furthermore, Hsp27 inhibited CD40 ligand production in astrocytes. Interestingly, IL- 8 is a proinflammatory cytokine and TGF- $\beta_{1}$ can act as an antiinflammatory factor by inhibition of local inflammation resulting in reduced tissue injury that may occur as a response to inflammation (Wahl, 1992). Furthermore, the interaction of CD40 ligand with its receptor has been implicated in the modulation of anti-inflammatory responses (Liu et al., 2010). Thus, Hsp27 may exert a proinflammatory effect, both through inducing a proinflammatory reaction (e.g., IL-8) and reducing the production of the anti-inflammatory factors (e.g., CD40 ligand and TGF- $\beta_{1}$ ) (Liu et al., 2010). In addition, Hsp27 may also function 

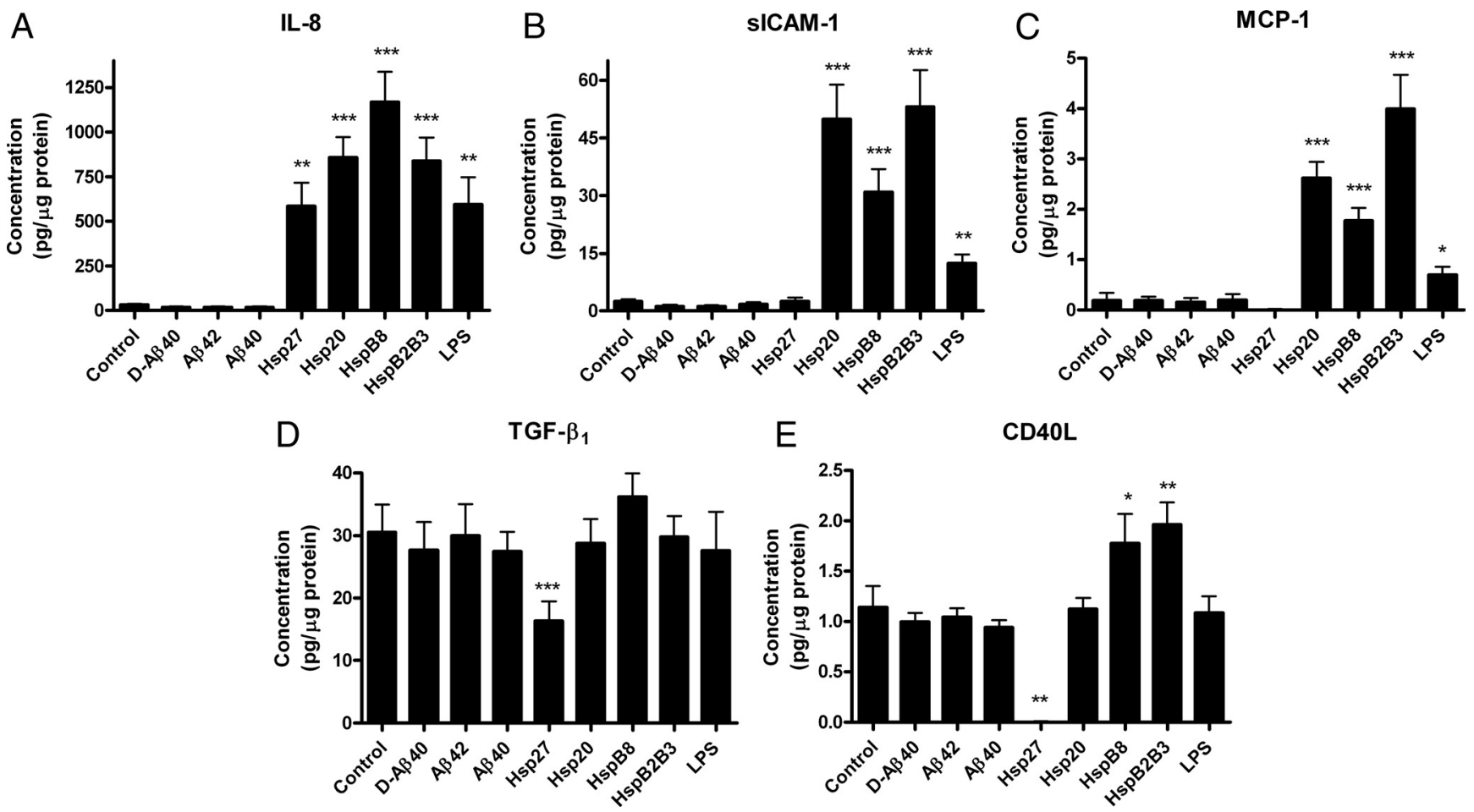

Figure 5. Production of inflammatory factors by human brain astrocytes. Cultured astrocytes were incubated with $12.5 \mu \mathrm{m} \mathrm{D}-\mathrm{A} \beta_{40}, \mathrm{~A} \beta_{42}, \mathrm{~A} \beta_{40}, \mathrm{Hsp27}, \mathrm{Hsp20}, \mathrm{HspB8}, \mathrm{HspB2B} 3$, or $1 \mu \mathrm{g} / \mathrm{ml} \mathrm{LPS}$ for $2 \mathrm{~d}$ at $37^{\circ}$. Supernatant was collected and IL-8 (A), sICAM-1 (B), MCP-1 (C), TGF- $\beta_{1}(\boldsymbol{D})$, and CD40 ligand (E) concentrations were measured using ELISA. Statistical analysis was performed using an independent sample $t$ test. The level of significance of the difference compared with control is indicated as follows: ${ }^{*} p<0.05 ;{ }^{* *} p<0.01 ;{ }^{* * *} p<0.001 ; p>0.05$ is not indicated. Means \pm SEM are shown.

as an indirect anti-apoptotic molecule (Concannon et al., 2003) by inhibiting the proapoptotic proteins caspase-3 (Stetler et al., 2009) and Bax (Havasi et al., 2008). Since both TGF- $\beta_{1}$ and CD40 ligand may be involved in apoptosis during inflammation (Calingasan et al., 2002; Schuster and Krieglstein, 2002), reduced TGF $\beta 1$ and CD40 ligand levels induced by Hsp27 may add to the anti-apoptotic effect of Hsp27. Furthermore, in transgenic mice models for $\mathrm{AD}$ it has been demonstrated that TGF- $\beta_{1}$ overexpression may lead to $\mathrm{A} \beta$ deposits in cerebral blood vessels and meningeal vessels (Wyss-Coray et al., 1997). Moreover, elevated levels of CD40 ligand may lead to endothelial dysfunction and atherothrombosis, whereas inhibition of CD40/CD40 ligand interaction may prevent atherogenesis in animal models (Urbich and Dimmeler, 2004; Chakrabarti et al., 2010; Dominguez-Rodriguez et al., 2010; Yoshioka et al., 2010). Thus, since Hsp27 affects the expression of TGF- $\beta_{1}$ and CD40 ligand, Hsp27 may create a proinflammatory environment, likely both by reducing the production of anti-inflammatory factors and inducing production of proinflammatory cytokines, inhibit apoptotic processes and may alter the vascular micro-environment. However, more study is required to elucidate the exact role of Hsp27 in neurovascular inflammation in $\mathrm{AD}$.

It has been suggested that $A \beta$ itself can induce neuroinflammation (Heneka et al., 2010). In our study, however, $A \beta_{1-40}$, $\mathrm{A} \beta_{1-42}$, and $\mathrm{D}-\mathrm{A} \beta_{1-40}$ had no effect on the production of inflam-

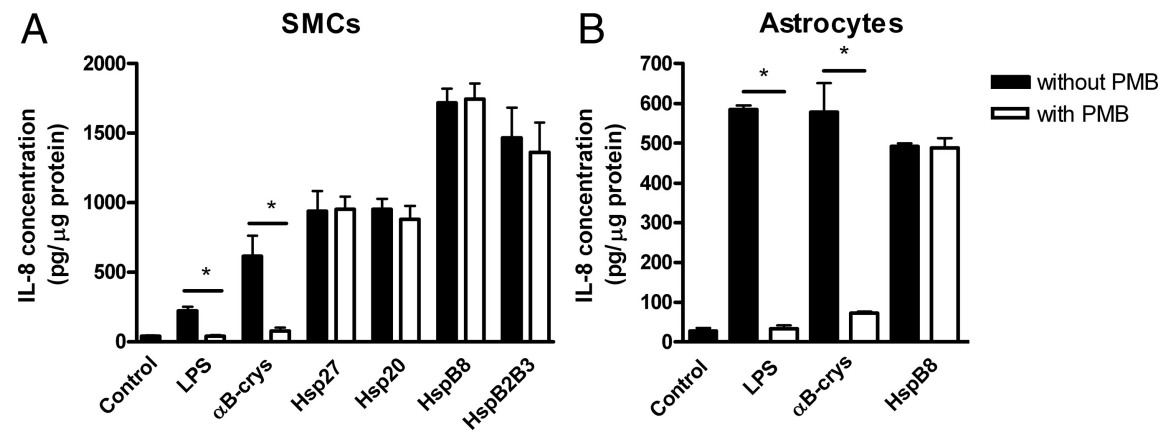

Figure 6. Production of IL-8 by human brain leptomeningeal SMCs $(\boldsymbol{A})$ and human brain astrocytes $(\boldsymbol{B})$ after coincubation with $\mathrm{PMB}$ Cultured cells were coincubated with $1 \mu \mathrm{g} / \mathrm{ml}$ LPS or a $12.5 \mu$ m concentration of the indicated sHsp with or without $10 \mu \mathrm{g} / \mathrm{ml} \mathrm{PMB} \mathrm{for} 2 \mathrm{~d}$ at $37^{\circ} \mathrm{C}$. In these experiments, PMB was preincubated with both the LPS and the sHsps for $1 \mathrm{~h}$ at $37^{\circ} \mathrm{C}$ before addition to the cells. Supernatant was collected and IL-8 concentrations were measured using ELISA. PMB efficiently abolished the effects of LPS and $\alpha \mathrm{B}-$ crystallin on IL-8 production, but not of the other $\mathrm{sHsps}$. Statistical analysis was performed using an independent sample $t$ test. The level of significance of the difference compared with control is indicated as follows: ${ }^{*} p<0.05 ; p>0.05$ is not indicated. Means \pm SEM are shown.

matory factors by SMCs and astrocytes. Previously, we also demonstrated that $\mathrm{A} \beta$ did not induce IL- 6 production by cerebrovascular cells and astrocytes (Wilhelmus et al., 2009). However, in the same study it was shown that $A \beta$ slightly induced IL- 6 production by microglia (Wilhelmus et al., 2009). In addition, in another study dose-dependent increases of IL- 8 and MCP- 1 production by human brain microglia were observed after exposure to $\mathrm{A} \beta$ (Lue et al., 2001). Together, these studies suggest that in the brain, microglial cells may be particularly susceptible to $A \beta$, resulting in an inflammatory reaction, but that other cerebral cell types, such as vascular cells and astrocytes, do not respond to $\mathrm{A} \beta$ in a proinflammatory way, but may be stimulated to do so by sHsps. 
In conclusion, Hsp20, HspB8 and $\mathrm{HspB} 2$ are associated with (cap)CAA and dyshoric angiopathy in $\mathrm{AD}$ brains with severe CAA pathology. In addition, colocalization of these sHsps with ICAM- 1 around $\mathrm{A} \beta$-laden vessels was observed. Furthermore, we demonstrated that Hsp20, HspB8 and HspB2B3 induced the production of inflammatory factors in vitro, suggesting that these sHsps might be among the key mediators of the inflammatory reactions associated with (cap)CAA and dyshoric angiopathy. An interesting role for Hsp27 was found, since this sHsp seems to induce a proinflammatory effect by reducing the normal antiinflammatory effect in human SMC and astrocytes in vitro. Furthermore, although it has been suggested that $\mathrm{A} \beta$ itself can induce inflammation (Heneka et al., 2010), we did not observe such an effect in cultured SMCs and astrocytes. The results of this study provide new insights in the inflammatory reactions associated with $\mathrm{AD}$ and $\mathrm{CAA}$ and, therefore, might provide interesting new targets for therapeutic intervention in the pathogenesis of $\mathrm{AD}$ and CAA.

\section{References}

Akiyama H, Barger S, Barnum S, Bradt B, Bauer J, Cole GM, Cooper NR, Eikelenboom P, Emmerling M, Fiebich BL, Finch CE, Frautschy S, Griffin WS, Hampel H, Hull M, Landreth G, Lue L, Mrak R, Mackenzie IR, McGeer PL, et al. (2000) Inflammation and Alzheimer's disease. Neurobiol Aging 21:383-421.

Attems J, Jellinger KA (2004) Only cerebral capillary amyloid angiopathy correlates with Alzheimer pathology—a pilot study. Acta Neuropathol 107:83-90.

Attems J, Jellinger K, Thal DR, Van Nostrand W (2011) Sporadic cerebral amyloid angiopathy*. Neuropathol Appl Neurobiol 37:75-93.

Braak H, Braak E (1991) Neuropathological stageing of Alzheimer-related changes. Acta Neuropathol 82:239-259.

Bruinsma IB, Wilhelmus MM, Kox M, Veerhuis R, de Waal RM, Verbeek MM (2010) Apolipoprotein E protects cultured pericytes and astrocytes from D-Abeta(1-40)-mediated cell death. Brain Res 1315C:169-180.

Calingasan NY, Erdely HA, Altar CA (2002) Identification of CD40 ligand in Alzheimer's disease and in animal models of Alzheimer's disease and brain injury. Neurobiol Aging 23:31-39.

Chakrabarti S, Rizvi M, Morin K, Garg R, Freedman JE (2010) The role of CD40L and VEGF in the modulation of angiogenesis and inflammation. Vascul Pharmacol 53:130-137.

Chung KK, Anderson NE, Hutchinson D, Synek B, Barber PA (2011) Cerebral amyloid angiopathy related inflammation: three case reports and a review. J Neurol Neurosurg Psychiatry 82:20-26.

Concannon CG, Gorman AM, Samali A (2003) On the role of Hsp27 in regulating apoptosis. Apoptosis 8:61-70.

Davis J, Van Nostrand WE (1996) Enhanced pathologic properties of Dutch-type mutant amyloid beta-protein. Proc Natl Acad Sci U S A 93:2996-3000.

De Groot CJ, Langeveld CH, Jongenelen CA, Montagne L, Van Der Valk P, Dijkstra CD (1997) Establishment of human adult astrocyte cultures derived from postmortem multiple sclerosis and control brain and spinal cord regions: immunophenotypical and functional characterization. J Neurosci Res 49:342-354.

den Engelsman J, Keijsers V, de Jong WW, Boelens WC (2003) The small heat-shock protein alpha B-crystallin promotes FBX4-dependent ubiquitination. J Biol Chem 278:4699-4704.

Dominguez-Rodriguez A, Abreu-Gonzalez P, Avanzas P, Gomez MA, Kaski JC (2010) Elevated circulating soluble form of CD40 ligand in patients with cardiac syndrome X. Atherosclerosis 213:637-641.

Eng JA, Frosch MP, Choi K, Rebeck GW, Greenberg SM (2004) Clinical manifestations of cerebral amyloid angiopathy-related inflammation. Ann Neurol 55:250-256.

Eurelings LS, Richard E, Carrano A, Eikelenboom P, van Gool WA, Rozemuller AJ (2010) Dyshoric capillary cerebral amyloid angiopathy mimicking Creutzfeldt-Jakob disease. J Neurol Sci 295:131-134.

Fan R, Xu F, Previti ML, Davis J, Grande AM, Robinson JK, Van Nostrand WE (2007) Minocycline reduces microglial activation and improves behavioral deficits in a transgenic model of cerebral microvascular amyloid. J Neurosci 27:3057-3063.
Harning R, Mainolfi E, Bystryn JC, Henn M, Merluzzi VJ, Rothlein R (1991) Serum levels of circulating intercellular adhesion molecule 1 in human malignant melanoma. Cancer Res 51:5003-5005.

Havasi A, Li Z, Wang Z, Martin JL, Botla V, Ruchalski K, Schwartz JH, Borkan SC (2008) Hsp27 inhibits Bax activation and apoptosis via a phosphatidylinositol 3-kinase-dependent mechanism. J Biol Chem 283:12305-12313.

Heneka MT, O'Banion MK, Terwel D, Kummer MP (2010) Neuroinflammatory processes in Alzheimer's disease. J Neural Transm 117:919-947.

Kinnecom C, Lev MH, Wendell L, Smith EE, Rosand J, Frosch MP, Greenberg SM (2007) Course of cerebral amyloid angiopathy-related inflammation. Neurology 68:1411-1416.

Liu J, Hong S, Feng Z, Xin Y, Wang Q, Fu J, Zhang C, Li G, Luo L, Yin Z (2010) Regulation of lipopolysaccharide-induced inflammatory response by heat shock protein 27 in THP-1 cells. Cell Immunol 264:127-134.

Lue LF, Rydel R, Brigham EF, Yang LB, Hampel H, Murphy GM Jr, Brachova L, Yan SD, Walker DG, Shen Y, Rogers J (2001) Inflammatory repertoire of Alzheimer's disease and nondemented elderly microglia in vitro. Glia 35:72-79.

Malm T, Koistinaho M, Muona A, Magga J, Koistinaho J (2010) The role and therapeutic potential of monocytic cells in Alzheimer's disease. Glia 58:889-900.

McGeer PL, McGeer EG (1995) The inflammatory response system of brain: implications for therapy of Alzheimer and other neurodegenerative diseases. Brain Res Brain Res Rev 21:195-218.

Mirra SS, Heyman A, McKeel D, Sumi SM, Crain BJ, Brownlee LM, Vogel FS, Hughes JP, van Belle G, Berg L (1991) The Consortium to Establish a Registry for Alzheimer's Disease (CERAD). Part II. Standardization of the neuropathologic assessment of Alzheimer's disease. Neurology 41:479-486.

Renkawek K, Bosman GJ, Gaestel M (1993) Increased expression of heatshock protein $27 \mathrm{kDa}$ in Alzheimer disease: a preliminary study. Neuroreport 5:14-16.

Renkawek K, Bosman GJ, de Jong WW (1994) Expression of small heatshock protein hsp 27 in reactive gliosis in Alzheimer disease and other types of dementia. Acta Neuropathol 87:511-519.

Richard E, Carrano A, Hoozemans JJ, van Horssen J, van Haastert ES, Eurelings LS, de Vries HE, Thal DR, Eikelenboom P, van Gool WA, Rozemuller AJ (2010) Characteristics of dyshoric capillary cerebral amyloid angiopathy. J Neuropathol Exp Neurol 69:1158-1167.

Rothlein R, Mainolfi EA, Czajkowski M, Marlin SD (1991) A form of circulating ICAM-1 in human serum. J Immunol 147:3788-3793.

Rozemuller AJ, van Gool WA, Eikelenboom P (2005) The neuroinflammatory response in plaques and amyloid angiopathy in Alzheimer's disease: therapeutic implications. Curr Drug Targets CNS Neurol Disord 4:223-233.

Schuster N, Krieglstein K (2002) Mechanisms of TGF-beta-mediated apoptosis. Cell Tissue Res 307:1-14

Selkoe DJ (1991) The molecular pathology of Alzheimer's disease. Neuron 6:487-498

Sokolova A, Hill MD, Rahimi F, Warden LA, Halliday GM, Shepherd CE (2009) Monocyte chemoattractant protein-1 plays a dominant role in the chronic inflammation observed in Alzheimer's disease. Brain Pathol 19:392-398.

Stetler RA, Gao Y, Signore AP, Cao G, Chen J (2009) HSP27: mechanisms of cellular protection against neuronal injury. Curr Mol Med 9:863-872.

Sugiyama Y, Suzuki A, Kishikawa M, Akutsu R, Hirose T, Waye MM, Tsui SK, Yoshida S, Ohno S (2000) Muscle develops a specific form of small heat shock protein complex composed of MKBP/HSPB2 and HSPB3 during myogenic differentiation. J Biol Chem 275:1095-1104.

Tan J, Town T, Paris D, Mori T, Suo Z, Crawford F, Mattson MP, Flavell RA, Mullan M (1999) Microglial activation resulting from CD40-CD40L interaction after beta-amyloid stimulation. Science 286:2352-2355.

Urbich C, Dimmeler S (2004) CD40 and vascular inflammation. Can J Cardiol 20:681-683.

van de Klundert FA, Smulders RH, Gijsen ML, Lindner RA, Jaenicke R, Carver JA, de Jong WW (1998) The mammalian small heat-shock protein Hsp20 forms dimers and is a poor chaperone. Eur J Biochem 258:1014-1021.

van der Wal EA, Gómez-Pinilla F, Cotman CW (1993) Transforming growth factor-beta 1 is in plaques in Alzheimer and Down pathologies. Neuroreport 4:69-72. 
Veerhuis R, Janssen I, Hoozemans JJ, De Groot CJ, Hack CE, Eikelenboom P (1998) Complement C1-inhibitor expression in Alzheimer's disease. Acta Neuropathol 96:287-296.

Verbeek MM, Otte-Höller I, Westphal JR, Wesseling P, Ruiter DJ, de Waal RM (1994a) Accumulation of intercellular adhesion molecule-1 in senile plaques in brain tissue of patients with Alzheimer's disease. Am J Pathol 144:104-116.

Verbeek MM, Otte-Höller I, Wesseling P, Ruiter DJ, de Waal RM (1994b) Induction of alpha-smooth muscle actin expression in cultured human brain pericytes by transforming growth factor-beta 1 . Am J Pathol 144:372-382.

Verbeek MM, Otte-Höller I, Wesseling P, Ruiter DJ, de Waal RM (1996) Differential expression of intercellular adhesion molecule-1 (ICAM-1) in the A beta-containing lesions in brains of patients with dementia of the Alzheimer type. Acta Neuropathol 91:608-615.

Verbeek MM, de Waal RM, Schipper JJ, Van Nostrand WE (1997) Rapid degeneration of cultured human brain pericytes by amyloid beta protein. J Neurochem 68:1135-1141.

Verschuure P, Tatard C, Boelens WC, Grongnet JF, David JC (2003) Expression of small heat shock proteins HspB2, HspB8, Hsp20 and cvHsp in different tissues of the perinatal developing pig. Eur J Cell Biol 82:523-530.

Vinters HV, Wang ZZ, Secor DL (1996) Brain parenchymal and microvascular amyloid in Alzheimer's disease. Brain Pathol 6:179-195.

Wahl SM (1992) Transforming growth factor beta (TGF-beta) in inflammation: a cause and a cure. J Clin Immunol 12:61-74.

Wilhelmus MM, Boelens WC, Otte-Höller I, Kamps B, de Waal RM, Verbeek MM (2006a) Small heat shock proteins inhibit amyloid-beta protein aggregation and cerebrovascular amyloid-beta protein toxicity. Brain Res 1089:67-78.

Wilhelmus MM, Boelens WC, Otte-Höller I, Kamps B, Kusters B, MaatSchieman ML, de Waal RM, Verbeek MM (2006b) Small heat shock protein HspB8: its distribution in Alzheimer's disease brains and its inhibition of amyloid-beta protein aggregation and cerebrovascular amyloidbeta toxicity. Acta Neuropathol 111:139-149.

Wilhelmus MM, Otte-Höller I, Wesseling P, de Waal RM, Boelens WC, Verbeek MM (2006c) Specific association of small heat shock proteins with the pathological hallmarks of Alzheimer's disease brains. Neuropathol Appl Neurobiol 32:119-130.

Wilhelmus MM, de Waal RM, Verbeek MM (2007) Heat shock proteins and amateur chaperones in amyloid-Beta accumulation and clearance in Alzheimer's disease. Mol Neurobiol 35:203-216.

Wilhelmus MM, Boelens WC, Kox M, Maat-Schieman ML, Veerhuis R, de Waal RM, Verbeek MM (2009) Small heat shock proteins associated with cerebral amyloid angiopathy of hereditary cerebral hemorrhage with amyloidosis (Dutch type) induce interleukin-6 secretion. Neurobiol Aging 30:229-240.

Wyss-Coray T, Masliah E, Mallory M, McConlogue L, Johnson-Wood K, Lin C, Mucke L (1997) Amyloidogenic role of cytokine TGF-betal in transgenic mice and in Alzheimer's disease. Nature 389:603-606.

Xu F, Grande AM, Robinson JK, Previti ML, Vasek M, Davis J, Van Nostrand WE (2007) Early-onset subicular microvascular amyloid and neuroinflammation correlate with behavioral deficits in vasculotropic mutant amyloid beta-protein precursor transgenic mice. Neuroscience 146:98-107.

Yamada M, Itoh Y, Shintaku M, Kawamura J, Jensson O, Thorsteinsson L, Suematsu N, Matsushita M, Otomo E (1996) Immune reactions associated with cerebral amyloid angiopathy. Stroke 27:1155-1162.

Yoshioka T, Funayama H, Hoshino H, Nakamura T, Sugawara Y, Kubo N, Nagata O, Ako J, Ishikawa SE, Kawakami M, Momomura S (2010) Association of CD40 ligand levels in the culprit coronary arteries with subsequent prognosis of acute myocardial infarction. Atherosclerosis 213: $268-272$. 